Published in final edited form as:

Adv Exp Med Biol. 2015 ; 889: 105-118. doi:10.1007/978-3-319-23730-5_7.

\title{
microRNAs and Prostate Cancer
}

\author{
Sajni Josson, \\ Uro-Oncology Research Program, Department of Medicine, Samuel Oschin Comprehensive \\ Cancer Institute, Cedars-Sinai Medical Center, Los Angeles, CA 90048, USA \\ Neostrata Inc., Princeton, NJ 08540, USA \\ Leland W. K. Chung, and \\ Uro-Oncology Research Program, Department of Medicine, Samuel Oschin Comprehensive \\ Cancer Institute, Cedars-Sinai Medical Center, Los Angeles, CA 90048, USA

\section{Murali Gururajan} \\ Uro-Oncology Research Program, Department of Medicine, Samuel Oschin Comprehensive \\ Cancer Institute, Cedars-Sinai Medical Center, Los Angeles, CA 90048, USA \\ Bristol-Myers Squibb Inc., Princeton, NJ 08543, USA \\ Sajni Josson: sajni.j@gmail.com; Leland W. K. Chung: Leland.Chung@cshs.org; Murali Gururajan: \\ gururajanmurali@gmail.com
}

\section{Abstract}

microRNAs are noncoding RNAs that are important for embryonic stem cell development and epithelial to mesenchymal transition (EMT). Tumor cells hijack EMT and stemness to grow and metastasize to distant organs including bone. In the tumor microenvironment, tumor cells interact with the stromal fibroblasts at the primary and metastatic sites and this interaction leads to tumor growth, EMT, and bone metastasis. Tumor-stromal interactions are a dynamic process that involves both cell-cell communications and extracellular vesicles and soluble factors. Growing body of evidence suggests that microRNAs are part of the payload that comprises the extracellular vesicles. microRNAs induce reactive stroma and thus convert normal stroma into tumorassociated stroma to promote aggressive tumorigenicity in vitro and in vivo. Landmark published studies demonstrate that expression of specific microRNAs of DLK1-DIO3 stem cell cluster correlates with patient survival in metastatic prostate cancer. Thus, microRNAs mediate tumor growth, EMT, and metastasis through cell intrinsic mechanisms and extracellular communications and could be novel biomarkers and therapeutic targets in bone metastatic prostate cancer.

\section{Keywords}

microRNA; Prostate cancer; DLK1-DIO3 cluster; EMT; Extracellular vesicles 


\section{Introduction}

Cancer cells metastasize to distant organs by hijacking of embryonic processes such as EMT and through intercellular communication via secretomes. The role of noncoding RNAs in EMT and metastasis of cancer cells remains poorly understood. Recent studies highlight the directive functions of noncoding RNAs including microRNAs (miRNAs) and long noncoding RNAs (lncRNAs) in modulating cancer cell growth, survival, EMT, and metastasis [1-5]. In contrast to lncRNAs, miRNAs are small ( 20-23 nucleotides) noncoding ribonucleic acid (RNA) molecules that bind to complementary sites in the messenger RNAs (mRNAs) of their target genes, thereby inducing the posttranscriptional silencing of genes [6]. It is predicted that miRNAs might regulate up to one-third of all genes. The presumed number of unidentified miRNAs is large. Currently, about 1881 miRNAs are annotated in Homo sapiens (human) based on miRBase. MiRNAs are located throughout the genome including intergenic regions and in the introns of both proteincoding and noncoding genes. Intronic miRNAs are primarily expressed with their host gene expression. The nonintronic miRNA encoding sequences are clustered at distinct genomic positions and are often coexpressed as a single polycistronic transcript.

\section{Role of miRNA in the Epigenetic Reprogramming of Prostate Cancer Metastasis}

DLK1-DIO3 cluster in prostate cancer bone metastasis: One of the largest miRNA clusters in the genome is on human chromosome 14q32 [7]. The delta-like 1 homolog-deiodinase, iodothyronine 3 (DLK1-DIO3) contains about $10 \%$ of the miRNAs currently known in mouse and human. This cluster is located within a well-known maternally imprinted region that is characterized by mono-allelic expression of the encompassed genes [7].

Evidence suggests deregulated expression of DLK1-DIO3 miRNAs and lncRNAs in cancer progression and metastasis $[8,9]$. Key miRNAs in this cluster including miR-409-3p/-5p, miR-154*, and miR-379 have been shown to be upregulated in prostate cancer and play a critical role in bone metastasis [10-12]. The DLK1-DIO3 miRNA members, known to affect embryonic development, were shown to be expressed by clinical prostate cancer specimens and actively participated in tumor-stromal interactions in cell and animal models of prostate cancer bone metastasis $[8,9,13,14]$.

The DLK1-DIO3 gene cluster was previously shown to be aberrantly silenced in humanand mouse-induced pluripotent stem cells (iPSCs) but not in fully pluripotent embryonic stem cells, indicating the importance in the generation of fully functional iPSCs [13, 14]. In these studies, miR-409-3p and miR-379 were expressed in embryonic cells and silenced in iPSC, which were not functional. In other studies compared mouse germline competent and incompetent cells and showed elevated levels of miR-379 and miR-409-5p in the germline competent cells compared to incompetent cells [13]. This suggests that these miRNAs are involved in embryogenesis and totipotency. The DLK1-DIO3 members appear to be activated during the metastatic process of cancer development. Several transgenic mouse models of prostate, liver and lung cancer also exhibit deregulated levels of DLK1-DIO3 cluster miRNA members [15-19]. Interestingly, miRNA members of the DLK1-DIO3 
cluster have been shown to be upregulated in the serum of cancer patients. Members of this cluster, miR-379, miR-154*, and miR-409-3p, show increased levels in the circulating exosomes of patients with prostate cancer [20], breast cancers [21], and lung adenocarcinomas [22].

Specifically, in prostate cancer, miR-409-3p has been shown to be upregulated in the serum of high-risk prostate cancer patients compared to low-risk prostate cancer patients [20]. Using MSKCC database it was demonstrated that miR-409-3p and miR-379 expression is strongly associated with progression-free survival of prostate cancer patients [10, 11]. It was also shown that miR-409-3p/-5p and miR-154* have higher expression in human prostate cancer tissues with higher Gleason $(\geq 7)$ score compared to benign prostatic hyperplasia using in situ hybridization and quantum dot analysis. Interestingly, increased staining of miR-409-3p/-5p and miR-154* expression was observed in prostate cancer bone metastatic tissue specimens. miR-379 expression was elevated in the tissues of metastatic prostate cancer compared to localized prostate cancer $[10,11]$. These studies demonstrate that some of the members of the DLK1-DIO3 cluster are elevated in both localized prostate cancer tissues, and metastatic prostate cancer tissues and in the serum of prostate cancer patients, and could be potential biomarkers for predicting the transition of indolent to aggressive form of prostate cancer.

\section{miR-409-3p/-5p}

miR-409-3p/-5p were shown to be elevated in prostate cancer tissues [11]. Previous studies demonstrated that miR-409 is tumorigenic in mouse models of prostate cancer. miR-409 was oncogenic when delivered orthotopically into intact mouse prostate gland using lentiviral miR-409 expressing plasmids. Overexpression of miR-409 led to transformation of normal mouse epithelial cells and prostatic hyperplasia and adenocarcinoma [11]. Thus, remarkably a single miRNA was shown to induce prostate cancer in mouse models. In prostate cancer cell models, overexpression of miR-409 led to decrease in expression of several tumor suppressor genes (Fig. 7.1).

Interestingly, miR-409-3p and miR-409-5p have distinct and shared target genes. Both miR-409-3p and miR-409-5p target Ras suppressor protein 1 (RSU1). RSU1 protein blocks the oncogenic Ras/MAPK pathway and integrin-linked kinase (ILK) pathway in prostate cancer [23-25]. miR-409-5p also targets stromal antigen 2 (STAG2). In the tumor cells, STAG2 is part of the cohesion complex. In cancer cells STAG2 is decreased and results in the deregulation of the cohesion complex, which is thought to cause aneuploidy, cancer initiation, and progression [26, 27]. miR-409-3p also targets key proteins such as polyhometic complex 3 (PHC3) and Von-Hippel-Lindau protein (VHL). PHC3 is part of the polycomb group complexes, involved in epigenetic reprogramming [28, 29]. Through analysis of MSKCC database, we observed that high miR-409-3p and low PHC3 expression correlated with disease-free survival of prostate cancer patients. Since PHC3 in the polycomb repressive complex 1 (PRC1) maintains the transcriptional repressive state of many genes and is critical for stem cell renewal, inhibition of PHC3 through miR-409-3p resulted in the reactivation of genes that promote cellular reprogramming and induce cancer stemness [28, 30]. 
The VHL protein is an ubiquitin ligase which ubiquitinates hypoxia-inducible factor-1a (HIF-1a) and marks it for proteasome degradation [31]. Thus, inhibition of VHL by miR-409-3p leads to activation of HIF-1a pathway. Interestingly, The DLK1-DIO3 cluster in embryogenesis is thought to be modulated by hypoxia [32]. The DLK1-DIO3 miRNA cluster is active in the early embryonic stages and is silenced subsequently [32]. Xie et al., compared initial-passage embryonic stem cells (ihESCs, $<10$ passages) and early passage ESC (ehESCs, 20-30 passages) from diverse cell types and demonstrated that in most of the cells DLK1-DIO3 cluster is silenced in ehESCs but not in ihESCs. They concluded that silencing of DLK1-DIO3 is triggered by high oxygen conditions (20\%) and this cluster is active only under low (5\%) oxygen conditions [32]. Additionally, miR-409 overexpression in prostate cancer cells also increased mitochondrial superoxide production by inhibition of mitochondrial manganese superoxide dismutase (Josson et al., unpublished observations).

Since combined miR-409-3p/-5p promote aneuploidy, activate oncogenic Ras, and hypoxiainduced signaling network through increased HIF-1a and elevated reactive oxygen species, this leads to the induction of EMT and cancer cell invasion and metastasis [10]. Consistent with these observations, inhibition of miR-409-5p in a human prostate cancer ARCaP EMT model we observed a reversal of ARCaP M to ARCaP E, or mesenchymal to epithelial transition (MET) and decreased cancer cell growth, invasion, and metastasis [11]. We demonstrated whereas in immunedeficient SCID mice, after inoculated with ARCaP M cells, $100 \%$ incidence of bone metastasis was observed, mice inoculated with ARCaP M cells with miR-409-5p knockdown prevented both tumor formation and tumor metastasis [11]. These results together suggest miR-409 plays a directive role conferring the development of aggressive prostate cancer and bone metastasis.

\section{$\operatorname{miR}-154^{\star}$ and $\mathrm{miR}-379$}

miR-154* is located adjacent to miR-409 within the long noncoding RNA, MEG9. miR-379 lies upstream of miR-154* in the DLK1-DIO3 cluster. Interestingly, consistent with the expression pattern of miR-409, miR-379 and miR-154* was also found to be elevated in prostate cancer human tissues [10]. Expression levels of miR-379 correlated with prostate cancer patient progression-free survival. miR-154* and miR-379 also promote EMT of prostate cancer cells. miR-154* was elevated in several isogenic prostate cancer ARCaP and LNCaP lines driving EMT and bone metastasis in mice. Additionally, miR-154* was elevated in embryonic stem cells. Inhibition of miR-154* in aggressive ARCaP M prostate cancer cells led to reversal of EMT with increased E-cadherin and decreased invasion in vitro [10]. Consistently, miR-154* inhibition also lead to reduced bone metastasis and increased survival in mice [10]. Mechanistically, miR-154* targets include tumor suppressors such as STAG2 and SMAD7. Decrease in STAG2 has been shown to induce aneuploidy in cancer cells [26, 27]. SMAD7 plays a critical role in inhibiting TGF- $\beta$ pathways [33] that lead to EMT [34]. miR-379 is also elevated in metastatic prostate cancer patients when compared to patients with localized disease [35].

In summary, overexpression of all four miRNA members (miR-409-3p, miR-409-5p, miR-154*, and miR-379) in nonmetastatic prostate cancer cells led to induction of EMT whereas inhibition of these cluster members led to the reversal of EMT in metastatic 
prostate cancer cells. Several targets such as STAG2 which is repressed by miR-409-5p and miR-154* and RSU1, targeted by miR-409-3p and miR-409-5p work synergistically to mediate the downstream effects. The oncogenic pathways that are activated in response to expression of this mega-cluster in cancer cells include, chromosomal aneuploidy, altered Ras-, HIF-1a-, TGF- $\beta-$, E2F-, Akt-, and polycomb-mediated signaling pathways [10]. Thus, members of this mega-cluster primarily function together to repress tumor suppressors and modulate the expression of genes involved in oncogenesis, EMT, and stemness to mediate downstream convergent signal axes.

\section{miR-145 and miR-143}

Unlike the imprinted clusters of miRNAs, miR-145 and miR-143 are significantly decreased in prostate cancer and in bone metastatic patients compared to normal prostate tissues [36]. miR-145 plays a critical role in EMT by targeting a master transcription regulator, ZEB2 [37]. Additionally, ZEB2 inhibits miR-145 forming a negative-feedback loop [37]. Other studies demonstrate that miR-145 targets mRNAs of ERG [38] and DNMT3b [39], whereas miR-143 targets ERK5 [40]. miR-145 is regulated through DNA methylation and p53 mutation pathways [41]. Overexpression of miR-145 and -143 decreased invasion and migration, with increased E-cadherin and decreased fibronectin. These cells underwent MET and had decreased prostate cancer bone metastasis [36]. Thus, downregulation of these miRNA are critical for EMT and prostate cancer bone metastasis and strongly predict disease-free survival [36, 37].

\section{Epigenetic Silencing by EZH2 Regulate miRNA}

Cao et al. demonstrated that several miRNAs were downregulated in prostate cancer, resulting in the activation of members of the polycomb group complexes [42]. The polycomb repressive complexes (PRC) include PRC1 and PRC2. These are involved in silencing of genes through histone modification and compaction of chromatin [43, 44]. The PRC2 complex methylates histones and allows for the binding of the PRC1 complex to the methylated site. PRC1 complex further ubiquinates histones and leads to compaction of chromatin. This prevents RNA Pol II activity and SWI-SNF accessibility to promoters leading to gene silencing [44].

\section{miR-200 cluster}

miR-200 cluster includes miR-200a, miR-200b, miR-200c, miR-141, and miR-429. Several of these miRNA are shown to be downregulated in human prostate cancer tissues compared to normal tissues $[45,46]$. While the steady-state levels of these miRNAs are upregulated in normal epithelial cells, downregulation of miR-200 members has shown to induce EMT and cancer metastasis in several cancer types [4, 47]. These miRNAs repress EMT master regulators such as ZEB1 and ZEB2 [48]. MiR-200 cluster members target PRC1 members of the epigenetic machinery [42]. Both, miR-200b and miR-200c repress RING2 of PRC1 complex and miR-200a, b target BMI1 [42]. miR-200b and miR-200c are also negatively regulated by PRC2 protein EZH2 (a histone methyl transferase). EZH2 is elevated in human prostate cancer tissues [49]. EZH2 protein downregulates miR-200b and miR-200c. Decreased miR-200 in turn derepresses PRC1 members resulting in activation of PRC1 
members and transcriptionally represses their target genes [42]. Overexpression of these miRNA in prostate cancer cells lead to decreased cell growth, invasion, decreased growth soft agar, and prostatosphere formation [42].

miR-181a and miR-181b

Similar to miR-200b,c, these miRNAs are also significantly downregulated in human prostate cancer tissue compared to normal [42]. These miRNA are also downregulated by EZH2 leading to activation of PRC1 member (RING2) and transcription repression of genes. Overexpression of these miRNA in prostate cancer cells blocked growth invasion and selfrenewal capacity of cancer cells [42].

miR-203

miR-203 expression is decreased in prostate cancer tissues compared to normal prostate. Several studies demonstrate the role of miR-203 in EMT and cancer metastasis [50, 51]. miR-203 target genes include, BMI1, Ras inhibitor (Rap1A) [52], LIM and SH3 domain protein 1 (LASP1, involved in dynamic actin-based cytoskeletal activities) [50] and SNAI2 [53]. These proteins are regulators of EMT and cancer metastasis. Overexpression of miR-203, as expected, suppressed prostate cancer bone metastasis through inhibition of invasion and migration and EMT. miR-203 was shown to downregulate pro-metastatic genes such as ZEB2, survivin, and Runx2, which are believed to be key master regulators of bone metastasis [51]. LASP, a target of miR-203 has been linked to metastatic disease in breast, colon, liver, and bladder cancer [50]. Negative-feedback action was shown between miR-203 and EZH2 [42]. Increased EZH2 in prostate cancer cells downregulated miR-203, which could potentially drive the migratory, invasive, and metastatic programs in prostate cancer cells [42].

miR-101

miR-101 was shown to be significantly downregulated in human prostate cancer tissues and serum compared to normal prostate $[49,54]$. It has been shown that one or two of the genomic loci encoding miR-101 is lost in one-third of the localized prostate cancer cells and in two-third of the metastatic disease cells [49]. miR-101 targets EZH2, and downregulation of miR-101 lead to activation of EZH2 expression levels in prostate cancer tissues. miR-101 appears to be upstream of miR-200b,c and miR-181a,b signaling [42, 55]. Several studies in different prostate cancer cells demonstrated that overexpression of miR-101 decreased cell proliferation in vitro [42, 56, 57]. Prostate cancer cells expressing miR-101 also had decreased tumor growth in xenograft mouse models [57, 58]. Upstream regulators of miR-101 include androgen signaling and HIF1a signaling [56].

\section{Role of Stromal miRNA in Prostate Cancer Development}

Aberrant tumor-stromal interactions in the tumor microenvironment could trigger cancer progression [59]. The stromal fibroblasts have been shown to drive cancer growth and metastasis [60]. In prostate cancer, the cancer-associated "reactive" stromal fibroblasts obtained from prostate cancer patients at the primary site and bone metastatic site (i.e., marrow stromal or osteosarcoma) accelerated prostate cancer growth, invasion, and 
metastasis in a highly cell contact-dependent manner, readily demonstrable in 3-D in vitro coculture or in vivo tumor models [59]. Tumor-stromal interactions resulted in the coevolution of prostate cancer and stroma which occurred under 3-D cocultured conditions. As a consequence of this co-evolution, the epithelium and stroma express shared biomarkers, which offer predictive value for prostate cancer invasion and metastasis [59, 61]. Several stroma-derived miRNA have been described to promote prostate tumor growth, EMT, stemness, and bone metastasis [12, 62, 63].

miRNA from the DLK1-DIO3 cluster: Interestingly, members of the DLK1-DIO3 cluster were highly expressed in cancer-associated stromal (CAS) fibroblasts derived from human prostate cancer patients. These miRNAs include miR-409-3p/-5p, miR-379, and miR-154* [12] (Fig. 7.2).

miR-409 was highly elevated in the CAS from both the prostate and the bone. MiR-409-3p and miR-409-5p expression analysis was performed using in situ hybridization and quantum dots multiplex labeling by employing human prostate cancer and normal prostate tissues [12]. Results of these studies demonstrated that miR-409-3p was significantly elevated in the stroma of patients with high Gleason score ( $\geq 7)$ compared to low Gleason $(<7)$. miR-409 was transduced and overexpressed in normal prostatic stromal cells. Interestingly, these stromal cells had morphologic features of myofibroblasts and became highly secretory [12].

The secretory microvesicles had elevated levels of miR-409-3p and miR-409-5p. miR-409-3p was significantly more abundant than miR-409-5p. Cancer cells, maintained in conditioned media collected from normal and miR-409 expressing prostate stromal cells, underwent EMT with an increase in miR-409-3p and miR-409-5p. These miRNA were taken up by the cancer cells, and downregulated miR-409-3p and miR-409-5p target genes such as STAG2, RSU1, and PHC3 in the cancer cells; decreased function of these tumor suppressors could be the underlying molecular basis leading to activation of EMT and oncogenic pathways in the cancer cells [12]. When the stroma cells expressing miR-409 were co-inoculated with prostate cancer cells (not expressing miR-409) in nude mice, they developed tumors with explosive growths. Stromal miR-409 was shown to be secreted into the microenvironment, taken up by the adjacent cancer cells, and directed cancer migration, invasion, and spread through the tumor areas. miR-409 release through extracellular vesicles resulted in tumor growth by inhibiting tumor suppressors such as RSU1 and STAG2 both in vitro and in vivo. Stromal miR-409 expression orchestrates secretion of miR-409-3p/-5p and other soluble factors, such as $\beta 2-\mathrm{M}$ which induces EMT and increases metastatic ability of prostate cancer cells [12].

Since miR-409 is activated during embryogenesis, we observed some of the stem cell markers (SOX2) upregulated in miR-409-expressing stromal cells. Stroma-derived miR-409 is capable of promoting tumor growth and EMT of adjacent tumor epithelia. Thus, miR-409 may be a new therapeutic target to break away the vicious cycle between stromal-epithelial interaction leading to EMT and prostate cancer bone and visceral organ metastases [12].

Recently, it was reported that breast cancer-associated exosomes contain pre-miRNAs, along with Dicer, argonaute protein (AGO2), and TAR (HIV-1) RNA Binding Protein 
(TRBP) $[64,65]$. This process was mediated by CD43 that promoted accumulation of Dicer specifically in cancer exosomes. Melo et al. demonstrated that cancer exosomes mediated an efficient and rapid silencing of mRNAs in the target cells to reprogram the target cell transcriptome [64]. Exosomes derived from cancer cells and serum of patients with breast cancer provoked nontumorigenic epithelial cells to form tumors in a Dicer-dependent manner [64]. It will be interesting to see if the miRNAs payload from exosomes can alter permanently the phenotype of nontumorigenic or bystander tumor cells and whether these communications, mediated by miRNAs, are targetable.

Musumeci et al., demonstrated that miR-15 and miR-16 are dowregulated in CAS. Decreased miR-15 and miR-16 resulted in increased tumor growth and progression through repression of FGF2 and FGFR1 signaling known to mediate tumorstroma interaction in prostate cancer [66]. Stromal-derived miR-21 was shown to predict biochemical recurrence in prostate cancer patients with Gleason grade 6 [67].

\section{Pathophysiological Relevance of DLK1-DIO3 Cluster microRNAs in Prostate Cancer and Its Implications for Prostate Cancer Biology, Biomarker Studies, and Therapy}

Since imprinted cluster of miRNAs are upregulated, the knockdown studies generally reveal more pathophysiologic relevant information than the downregulated miRNAs which has to be overexpressed to establish their role in cancer biology and metastasis (Table 7.1). In this context, the recent findings on the oncogenic and metastatic role for DLK1-DIO3 cluster miRNAs utilizing knockdown approaches establish the functions of specific miRNAs in the pathphysiology of prostate cancer and thus uncover potential biomarker and therapeutic targets for treating lethal bone metastatic prostate cancer.

Recent studies suggest another class of noncoding RNAs called lncRNAs in cancer development and progression. LncRNAs are more than 200 nucleotides long and regulate mRNAs by multiple mechanisms. However, unlike miRNAs, lncRNAs are not processed by the Dicer machinery and hence are not generated at equal lengths (unlike miRNAs which are 22 nucleotides in length) and have different mechanisms of target repression. However, increasing evidence suggests miRNAs and potentially lncRNAs helped pathologists diagnose and distinguish indolent from aggressive cancer. In addition to its role in biomarker evaluation, therapeutic targeting of miRNAs should sensitize prostate cancer cells to radiation therapy and chemotherapy.

\section{Conclusions}

Accumulating evidence suggest oncogenic and metastatic role for specific miRNAs of DLK1-DIO3 cluster in prostate cancer and that inhibition of cluster members led to reversal of EMT and reduced bone metastasis of prostate cancer. More importantly, elevated levels of miR-409 and miR-379 predict progression-free survival of patients, and thus promising potential biomarker for prostate cancer patients. DLK1-DIO3 cluster miRNAs have prognostic value in other cancer types including lung cancer. Future studies will need to explore large cohorts of patients for biomarker use of DLK1-DIO3 cluster miRNAs and 
small molecule approaches to target specific miRNAs of DLK1-DIO3 cluster to treat prostate cancer bone metastasis utilizing preclinical animal or 3-D growth models.

\section{Acknowledgments}

Grant support from PO1 CA098912.

\section{References}

1. Ma L, Teruya-Feldstein J, Weinberg RA. Tumour invasion and metastasis initiated by microRNA-10b in breast cancer. Nature. 2007; 449(7163):682-688. [PubMed: 17898713]

2. Tavazoie SF, Alarcon C, Oskarsson T, Padua D, Wang Q, Bos PD, et al. Endogenous human microRNAs that suppress breast cancer metastasis. Nature. 2008; 451(7175):147-152. [PubMed: 18185580]

3. Zhang Y, Yang P, Sun T, Li D, Xu X, Rui Y, et al. miR-126 and miR-126* repress recruitment of mesenchymal stem cells and inflammatory monocytes to inhibit breast cancer metastasis. Nat Cell Biol. 2013; 15(3):284-294. [PubMed: 23396050]

4. Korpal M, Ell BJ, Buffa FM, Ibrahim T, Blanco MA, Celia-Terrassa T, et al. Direct targeting of Sec23a by miR-200s influences cancer cell secretome and promotes metastatic colonization. Nat Med. 2011; 17(9):1101-1108. [PubMed: 21822286]

5. Chou J, Lin JH, Brenot A, Kim JW, Provot S, Werb Z. GATA3 suppresses metastasis and modulates the tumour microenvironment by regulating microRNA-29b expression. Nat Cell Biol. 2013; 15(2):201-213. [PubMed: 23354167]

6. Ambros V. microRNAs: tiny regulators with great potential. Cell. 2001; 107(7):823-826. [PubMed: 11779458]

7. Kircher M, Bock C, Paulsen M. Structural conservation versus functional divergence of maternally expressed microRNAs in the Dlk1/Gt12 imprinting region. BMC Genomics. 2008; 9:346. [PubMed: 18651963]

8. Hagan JP, O’Neill BL, Stewart CL, Kozlov SV, Croce CM. At least ten genes define the imprinted Dlk1-Dio3 cluster on mouse chromosome 12qF1. PLoS One. 2009; 4(2):e4352. [PubMed: 19194500]

9. Formosa A, Markert EK, Lena AM, Italiano D, Finazzi-Agro E, Levine AJ, et al. MicroRNAs, miR-154, miR-299-5p, miR-376a, miR-376c, miR-377, miR-381, miR-487b, miR-485-3p, miR-495 and miR-654-3p, mapped to the $14 \mathrm{q} 32.31$ locus, regulate proliferation, apoptosis, migration and invasion in metastatic prostate cancer cells. Oncogene. 2013; 33(44):5173-5182. [PubMed: 24166498]

10. Gururajan M, Josson S, Chu GC, Lu CL, Lu YT, Haga CL, et al. miR-154* and miR-379 in the DLK1-DIO3 microRNA mega-cluster regulate epithelial to mesenchymal transition and bone metastasis of prostate cancer. Clin Cancer Res. 2014; 20(24):6559-6569. [PubMed: 25324143]

11. Josson S, Gururajan M, Hu P, Shao C, Chu CY, Zhau HE, et al. miR-409-3p/-5p promotes tumorigenesis, epithelial to mesenchymal transition and bone metastasis of human prostate cancer. Clin Cancer Res. 2014; 20(17):4636-4646. [PubMed: 24963047]

12. Josson S, Gururajan M, Sung SY, Hu P, Shao C, Zhau HE, et al. Stromal fibroblast-derived miR-409 promotes epithelial-to-mesenchymal transition and prostate tumorigenesis. Oncogene. 2015; 34(21):2690-2699. [PubMed: 25065597]

13. Liu L, Luo GZ, Yang W, Zhao X, Zheng Q, Lv Z, et al. Activation of the imprinted Dlk1-Dio3 region correlates with pluripotency levels of mouse stem cells. J Biol Chem. 2010; 285(25): 19483-19490. [PubMed: 20382743]

14. Stadtfeld M, Apostolou E, Akutsu H, Fukuda A, Follett P, Natesan S, et al. Aberrant silencing of imprinted genes on chromosome $12 \mathrm{qF} 1$ in mouse induced pluripotent stem cells. Nature. 2010; 465(7295):175-181. [PubMed: 20418860]

15. Lim L, Balakrishnan A, Huskey N, Jones KD, Jodari M, Ng R, et al. MiR-494 within an oncogenic MicroRNA megacluster regulates G1/S transition in liver tumorigenesis through suppression of MCC. Hepatology. 2014; 59(1):202-215. [PubMed: 23913442] 
16. Luk JM, Burchard J, Zhang C, Liu AM, Wong KF, Shek FH, et al. DLK1-DIO3 genomic imprinted microRNA cluster at 14q32.2 defines a stemlike subtype of hepatocellular carcinoma associated with poor survival. J Biol Chem. 2011; 286(35):30706-30713. [PubMed: 21737452]

17. Haga CL, Phinney DG. MicroRNAs in the imprinted DLK1-DIO3 region repress the epithelial-tomesenchymal transition by targeting the TWIST1 protein signaling network. J Biol Chem. 2012; 287(51):42695-42707. [PubMed: 23105110]

18. Lempiainen H, Couttet P, Bolognani F, Muller A, Dubost V, Luisier R, et al. Identification of Dlk1-Dio3 imprinted gene cluster noncoding RNAs as novel candidate biomarkers for liver tumor promotion. Toxicol Sci. 2013; 131(2):375-386. [PubMed: 23091169]

19. Valdmanis PN, Roy-Chaudhuri B, Kim HK, Sayles LC, Zheng Y, Chuang CH, et al. Upregulation of the microRNA cluster at the Dlk1-Dio3 locus in lung adenocarcinoma. Oncogene. 2015; 34(1): 94-103. [PubMed: 24317514]

20. Nguyen HC, Xie W, Yang M, Hsieh CL, Drouin S, Lee GS, et al. Expression differences of circulating microRNAs in metastatic castration resistant prostate cancer and low-risk, localized prostate cancer. Prostate. 2013; 73(4):346-354. [PubMed: 22887127]

21. Li S, Meng H, Zhou F, Zhai L, Zhang L, Gu F, et al. MicroRNA-132 is frequently down-regulated in ductal carcinoma in situ (DCIS) of breast and acts as a tumor suppressor by inhibiting cell proliferation. Pathol Res Pract. 2013; 209(3):179-183. [PubMed: 23399321]

22. Cazzoli R, Buttitta F, Di Nicola M, Malatesta S, Marchetti A, Rom WN, et al. MicroRNAs derived from circulating exosomes as noninvasive biomarkers for screening and diagnosing lung cancer. $\mathrm{J}$ Thorac Oncol. 2013; 8(9):1156-1162. [PubMed: 23945385]

23. Gonzalez-Nieves R, Desantis AI, Cutler ML. Rsu1 contributes to regulation of cell adhesion and spreading by PINCH1-dependent and - independent mechanisms. J Cell Commun Signal. 2013; 7(4):273-279.

24. Dougherty GW, Jose C, Gimona M, Cutler ML. The Rsu-1-PINCH1-ILK complex is regulated by Ras activation in tumor cells. Eur J Cell Biol. 2008; 87(8-9):721-734. [PubMed: 18436335]

25. Becker-Santos DD, Guo Y, Ghaffari M, Vickers ED, Lehman M, Altamirano-Dimas M, et al. Integrin-linked kinase as a target for ERG-mediated invasive properties in prostate cancer models. Carcinogenesis. 2012; 33(12):2558-2567. [PubMed: 23027626]

26. Solomon DA, Kim T, Diaz-Martinez LA, Fair J, Elkahloun AG, Harris BT, et al. Mutational inactivation of STAG2 causes aneuploidy in human cancer. Science. 2011; 333(6045):1039-1043. [PubMed: 21852505]

27. Kim MS, Kim SS, Je EM, Yoo NJ, Lee SH. Mutational and expressional analyses of STAG2 gene in solid cancers. Neoplasma. 2012; 59(5):524-529. [PubMed: 22668012]

28. Deshpande AM, Akunowicz JD, Reveles XT, Patel BB, Saria EA, Gorlick RG, et al. PHC3, a component of the hPRC-H complex, associates with E2F6 during G0 and is lost in osteosarcoma tumors. Oncogene. 2007; 26(12):1714-1722. [PubMed: 17001316]

29. Iwata S, Takenobu H, Kageyama H, Koseki H, Ishii T, Nakazawa A, et al. Polycomb group molecule PHC3 regulates polycomb complex composition and prognosis of osteosarcoma. Cancer Sci. 2010; 101(7):1646-1652. [PubMed: 20491773]

30. Isono K, Fujimura Y, Shinga J, Yamaki M, O-Wang J, Takihara Y, et al. Mammalian polyhomeotic homologues Phc2 and Phc1 act in synergy to mediate polycomb repression of Hox genes. Mol Cell Biol. 2005; 25(15):6694-6706. [PubMed: 16024804]

31. Palayoor ST, Burgos MA, Shoaibi A, Tofilon PJ, Coleman CN. Effect of radiation and ibuprofen on normoxic renal carcinoma cells overexpressing hypoxia-inducible factors by loss of von Hippel-Lindau tumor suppressor gene function. Clin Cancer Res. 2004; 10(12 Pt 1):4158-4164. [PubMed: 15217953]

32. Xie P, Sun Y, Ouyang Q, Hu L, Tan Y, Zhou X, et al. Physiological oxygen prevents frequent silencing of the DLK1-DIO3 cluster during human embryonic stem cells culture. Stem Cells. 2014; 32(2):391-401. [PubMed: 24123616]

33. Edlund S, Bu S, Schuster N, Aspenstrom P, Heuchel R, Heldin NE, et al. Transforming growth factor-beta1 (TGF-beta)-induced apoptosis of prostate cancer cells involves Smad7-dependent activation of p38 by TGF-beta-activated kinase 1 and mitogen-activated protein kinase 3. Mol Biol Cell. 2003; 14(2):529-544. [PubMed: 12589052] 
34. Lenferink AE, Cantin C, Nantel A, Wang E, Durocher Y, Banville M, et al. Transcriptome profiling of a TGF-beta-induced epithelial-to-mesenchymal transition reveals extracellular clusterin as a target for therapeutic antibodies. Oncogene. 2010; 29(6):831-844. [PubMed: 19935703]

35. Bryant RJ, Pawlowski T, Catto JW, Marsden G, Vessella RL, Rhees B, et al. Changes in circulating microRNA levels associated with prostate cancer. Br J Cancer. 2012; 106(4):768-774. [PubMed: 22240788]

36. Peng X, Guo W, Liu T, Wang X, Tu X, Xiong D, et al. Identification of miRs-143 and-145 that is associated with bone metastasis of prostate cancer and involved in the regulation of EMT. PLoS One. 2011; 6(5):e20341. [PubMed: 21647377]

37. Ren D, Wang M, Guo W, Huang S, Wang Z, Zhao X, et al. Double-negative feedback loop between ZEB2 and miR-145 regulates epithelial-mesenchymal transition and stem cell properties in prostate cancer cells. Cell Tissue Res. 2014; 358(3):763-778. [PubMed: 25296715]

38. Hart M, Wach S, Nolte E, Szczyrba J, Menon R, Taubert H, et al. The proto-oncogene ERG is a target of microRNA miR-145 in prostate cancer. FEBS J. 2013; 280(9):2105-2116. [PubMed: 23480797]

39. Xue G, Ren Z, Chen Y, Zhu J, Du Y, Pan D, et al. A feedback regulation between miR-145 and DNA methyltransferase $3 b$ in prostate cancer cell and their responses to irradiation. Cancer Lett. 2015; 5

40. Clape C, Fritz V, Henriquet C, Apparailly F, Fernandez PL, Iborra F, et al. miR-143 interferes with ERK5 signaling, and abrogates prostate cancer progression in mice. PLoS One. 2009; 4(10):7542.

41. Suh SO, Chen Y, Zaman MS, Hirata H, Yamamura S, Shahryari V, et al. MicroRNA-145 is regulated by DNA methylation and p53 gene mutation in prostate cancer. Carcinogenesis. 2011; 32(5):772-778. [PubMed: 21349819]

42. Cao Q, Mani RS, Ateeq B, Dhanasekaran SM, Asangani IA, Prensner JR, et al. Coordinated regulation of polycomb group complexes through microRNAs in cancer. Cancer Cell. 2011; 20(2): 187-199. [PubMed: 21840484]

43. Bracken AP, Helin K. Polycomb group proteins: navigators of lineage pathways led astray in cancer. Nat Rev Cancer. 2009; 9(11):773-784. [PubMed: 19851313]

44. Di Croce L, Helin K. Transcriptional regulation by Polycomb group proteins. Nat Struct Mol Biol. 2013; 20(10):1147-1155. [PubMed: 24096405]

45. Barron N, Keenan J, Gammell P, Martinez VG, Freeman A, Masters JR, et al. Biochemical relapse following radical prostatectomy and miR-200a levels in prostate cancer. Prostate. 2012; 72(11): 1193-1199. [PubMed: 22161972]

46. Liu YN, Yin JJ, Abou-Kheir W, Hynes PG, Casey OM, Fang L, et al. MiR-1 and miR-200 inhibit EMT via Slug-dependent and tumorigenesis via Slug-independent mechanisms. Oncogene. 2013; 32(3):296-306. [PubMed: 22370643]

47. Gregory PA, Bert AG, Paterson EL, Barry SC, Tsykin A, Farshid G, et al. The miR-200 family and miR-205 regulate epithelial to mesenchymal transition by targeting ZEB1 and SIP1. Nat Cell Biol. 2008; 10(5):593-601. [PubMed: 18376396]

48. Guo F, Parker Kerrigan BC, Yang D, Hu L, Shmulevich I, Sood AK, et al. Post-transcriptional regulatory network of epithelial-to-mesenchymal and mesenchymal-to-epithelial transitions. J Hematol Oncol. 2014; 7:19. [PubMed: 24598126]

49. Varambally S, Cao Q, Mani RS, Shankar S, Wang X, Ateeq B, et al. Genomic loss of microRNA-101 leads to overexpression of histone methyltransferase EZH2 in cancer. Science. 2008; 322(5908):1695-1699. [PubMed: 19008416]

50. Hailer A, Grunewald TG, Orth M, Reiss C, Kneitz B, Spahn M, et al. Loss of tumor suppressor mir-203 mediates overexpression of LIM and SH3 Protein 1 (LASP1) in high-risk prostate cancer thereby increasing cell proliferation and migration. Oncotarget. 2014; 5(12):4144-4153. [PubMed: 24980827]

51. Saini S, Majid S, Yamamura S, Tabatabai L, Suh SO, Shahryari V, et al. Regulatory role of mir-203 in prostate cancer progression and metastasis. Clin Cancer Res. 2011; 17(16):5287-5298. [PubMed: 21159887] 
52. Xiang J, Bian C, Wang H, Huang S, Wu D. MiR-203 down-regulates Rap1A and suppresses cell proliferation, adhesion and invasion in prostate cancer. J Exp Clin Cancer Res. 2015; 34(1):8. [PubMed: 25636908]

53. Qu Y, Li WC, Hellem MR, Rostad K, Popa M, McCormack E, et al. MiR-182 and miR-203 induce mesenchymal to epithelial transition and self-sufficiency of growth signals via repressing SNAI2 in prostate cells. Int J Cancer. 2013; 133(3):544-555. [PubMed: 23354685]

54. Srivastava A, Goldberger H, Dimtchev A, Marian C, Soldin O, Li X, et al. Circulatory miR-628-5p is downregulated in prostate cancer patients. Tumour Biol. 2014; 35(5):4867-4873. [PubMed: 24477576]

55. Banerjee R, Mani RS, Russo N, Scanlon CS, Tsodikov A, Jing X, et al. The tumor suppressor gene rap1GAP is silenced by miR-101-mediated EZH2 overexpression in invasive squamous cell carcinoma. Oncogene. 2011; 30(42):4339-4349. [PubMed: 21532618]

56. Cao P, Deng Z, Wan M, Huang W, Cramer SD, Xu J, et al. MicroRNA-101 negatively regulates Ezh2 and its expression is modulated by androgen receptor and HIF-1alpha/HIF-1beta. Mol Cancer. 2010; 9:108. [PubMed: 20478051]

57. Hao Y, Gu X, Zhao Y, Greene S, Sha W, Smoot DT, et al. Enforced expression of miR-101 inhibits prostate cancer cell growth by modulating the COX-2 pathway in vivo. Cancer Prev Res. 2011; 4(7):1073-1083.

58. Ren G, Baritaki S, Marathe H, Feng J, Park S, Beach S, et al. Polycomb protein EZH2 regulates tumor invasion via the transcriptional repression of the metastasis suppressor RKIP in breast and prostate cancer. Cancer Res. 2012; 72(12):3091-3104. [PubMed: 22505648]

59. Sung SY, Hsieh CL, Law A, Zhau HE, Pathak S, Multani AS, et al. Coevolution of prostate cancer and bone stroma in three-dimensional coculture: implications for cancer growth and metastasis. Cancer Res. 2008; 68(23):9996-10003. [PubMed: 19047182]

60. Bhowmick NA, Chytil A, Plieth D, Gorska AE, Dumont N, Shappell S, et al. TGF-beta signaling in fibroblasts modulates the oncogenic potential of adjacent epithelia. Science. 2004; 303(5659): 848-851. [PubMed: 14764882]

61. Sung SY, Chung LW. Prostate tumor-stroma interaction: molecular mechanisms and opportunities for therapeutic targeting. Differentiation. 2002; 70(9-10):506-521. [PubMed: 12492493]

62. Gandellini P, Giannoni E, Casamichele A, Taddei ML, Callari M, Piovan C, et al. miR-205 hinders the malignant interplay between prostate cancer cells and associated fibroblasts. Antioxid Redox Signal. 2014; 20(7):1045-1059. [PubMed: 23924028]

63. Ren Q, Liang J, Wei J, Basturk O, Wang J, Daniels G, et al. Epithelial and stromal expression of miRNAs during prostate cancer progression. Am J Transl Res. 2014; 6(4):329-339. [PubMed: 25075250]

64. Melo SA, Sugimoto H, O'Connell JT, Kato N, Villanueva A, Vidal A, et al. Cancer exosomes perform cell-independent microRNA biogenesis and promote tumorigenesis. Cancer Cell. 2014; 26(5):707-721. [PubMed: 25446899]

65. Sato-Kuwabara Y, Melo SA, Soares FA, Calin GA. The fusion of two worlds: non-coding RNAs and extracellular vesicles_-diagnostic and therapeutic implications (Review). Int J Oncol. 2015; 46(1):17-27. [PubMed: 25338714]

66. Musumeci M, Coppola V, Addario A, Patrizii M, Maugeri-Sacca M, Memeo L, et al. Control of tumor and microenvironment cross-talk by miR-15a and miR-16 in prostate cancer. Oncogene. 2011; 30(41):4231-4242. [PubMed: 21532615]

67. Melbo-Jorgensen C, Ness N, Andersen S, Valkov A, Donnem T, Al-Saad S, et al. Stromal expression of MiR-21 predicts biochemical failure in prostate cancer patients with Gleason score 6. PLoS One. 2014; 9(11):e113039. [PubMed: 25401698] 


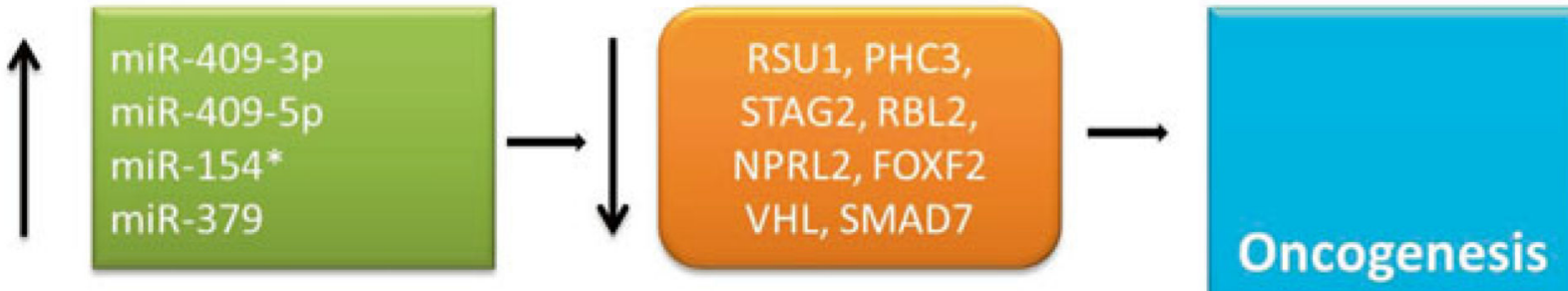

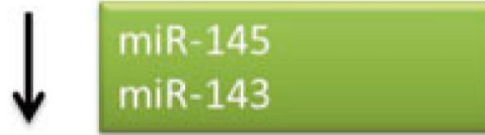

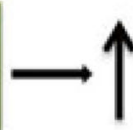

-<smiles>[AlH]</smiles>
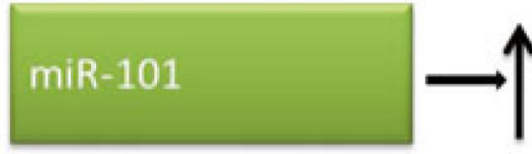

$\uparrow$
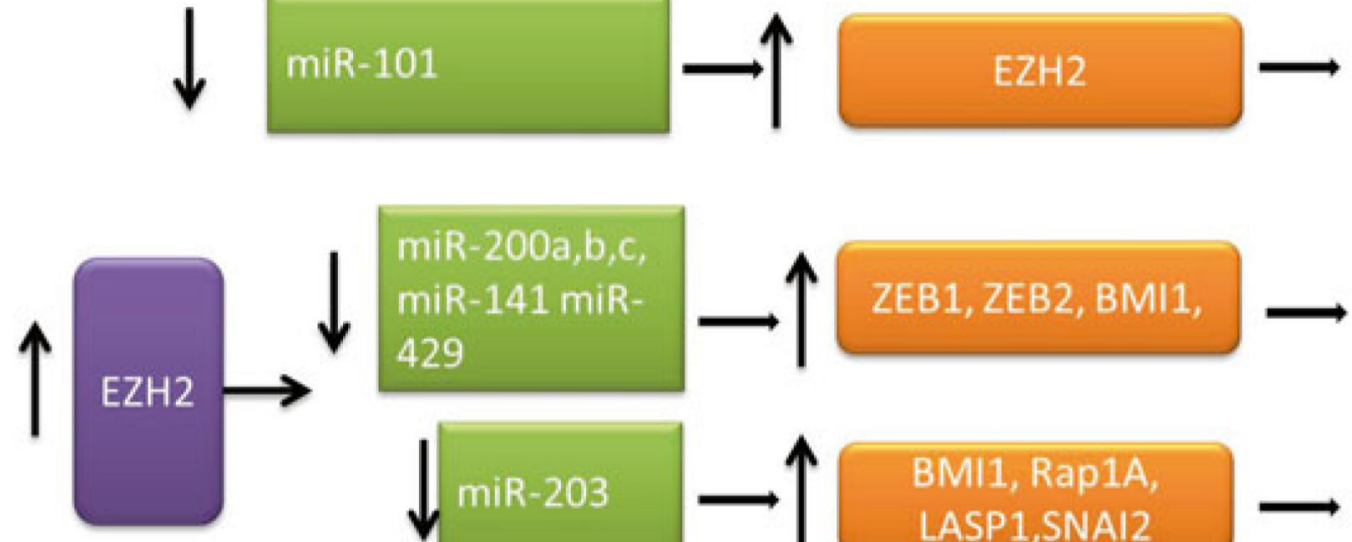

miR-200a,b,c, miR-141 miR429

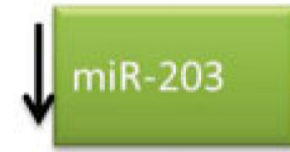

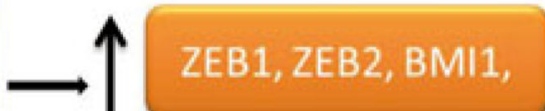<smiles>[13CH3]</smiles>

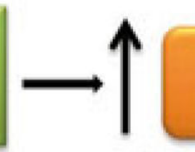

\section{BMI1, Rap1A,} LASP1,SNAI2

Fig. 7.1.

miRNA-mediated pathways in prostate cancer 

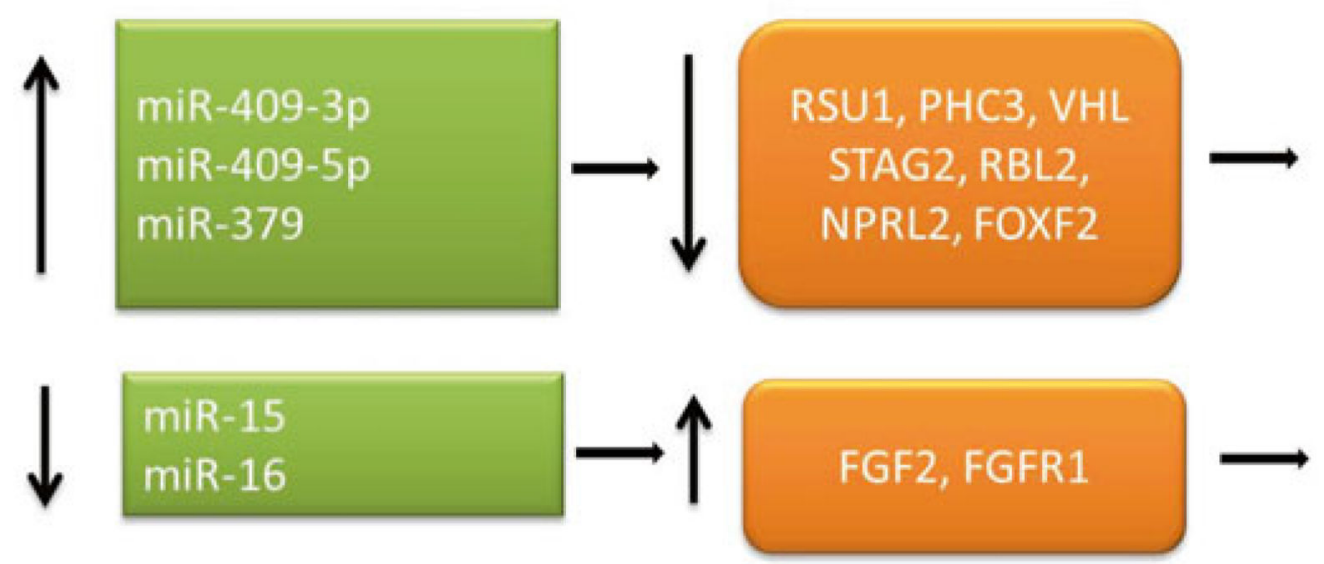

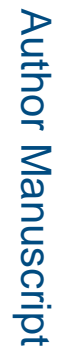

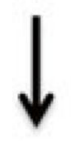

\section{miR-21}
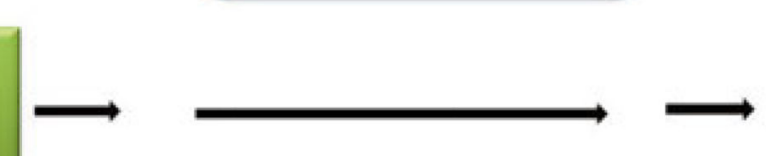

Fig. 7.2.

Stromal miRNA-mediated pathways in prostate cancer 
Table 7.1

Summary of microRNAs with a mechanistic role in prostate cancer

\begin{tabular}{lll}
\hline microRNAs in prostate cancer & Up/Downregulated & References \\
\hline miR-200a, b, c & Downregulated (tumor) & {$[45,46]$} \\
\hline miR-181a,b & Downregulated (tumor) & {$[53]$} \\
\hline miR-145 & Downregulated (tumor) & {$[38]$} \\
\hline miR-143 & Downregulated (tumor) & {$[36]$} \\
\hline miR-101 & Downregulated (tumor) & {$[56]$} \\
\hline miR-203 & Downregulated (tumor) & {$[50]$} \\
\hline miR-15, miR-16 & Downregulated (tumor/stroma) & {$[60]$} \\
\hline miR-21 & Upregulated (tumor/stroma) & {$[57]$} \\
\hline miR-409 & Upregulated (tumor/stroma) & {$[11,12]$} \\
\hline $\mathrm{miR}-379$ & Upregulated (tumor/stroma) & {$[10,12]$} \\
\hline $\mathrm{miR}-154 *$ & Upregulated (tumor/stroma) & {$[10,12]$} \\
\hline
\end{tabular}

